\title{
Spiel mir das Lied der Mortalitätsstatistik...
}

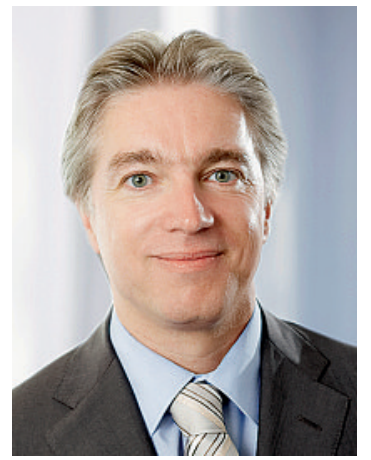

Daniel Herren
Man nehme eine Handvoll Routinedaten, vermische sie beliebig miteinander, würze sie mit einer Prise Ignoranz und fertig ist die Mortalitätsstatistik des Bundes.

Unter dem politischen Druck - seit Anfang Jahr verlangen der Artikel 22a des Krankenversicherungsgesetzes (KVG) und der Artikel 31 der dazugehörigen Krankenversicherungsver-

\section{Ungenügende Daten auf intransparenter Daten- basis sind schlechte Voraussetzungen für gültige und relevante Aussagen}

ordnung (KVV) unter anderem die Publikation von Qualitätsdaten - macht es sich das Bundesamt für Gesundheit (BAG) leicht und publiziert nun Mortalitätszahlen, die in dieser Form nicht haltbar sind. Das BAG beruft sich dabei auf Routinedaten des Bundesamtes für Statistik, die in den Spitälern erhoben wurden - jedoch zu ganz anderen statistischen Zwecken. Auf dieser zweifelhaften Grundlage schafft das BAG so einen Qualitätsindikator, der zum Quervergleich der Spitäler herangezogen wird.

Obwohl die Ärzteschaft wie auch die Spitäler massive Kritik an diesem Zahlenmaterial geübt haben, soll es nun unter dem Vorwand einer verbesserten Leistungstransparenz breit publiziert werden. Gegen Transparenz und Vergleichbarkeit ärztlicher Leistungen ist nichts einzuwenden. Das Tragische an den Publikationsabsichten des BAG besteht darin, dass wirklich niemand von der Veröffentlichung dieser Zahlen profitiert: Die Leistungserbringer sind so schlau wie zuvor, die Kassen hoffen vergebens auf die diskriminierende Variable, die den Spreu vom Weizen trennen soll, und die Patienten haben keinen Informationsgewinn, da die Zahlen nichts aussagen.

Ungenügende Daten auf einer intransparenten Datenbasis sind schlechte Voraussetzungen für gültige und relevante Aussagen. Solange keine Risikoanpassung vorgenommen wird, solange Tracer-Operationen gewählt werden, bei denen die Mortalität kein Qualitätskriterium darstellt, und solange die Datenqualität - und vor allem auch die Datenquantität - nicht kritisch hinterfragt und geprüft werden - solange wird die Diskussion um Qualitätsunterschiede in den verschiedenen Spitälern keine Verbesserung erfahren. Im Gegenteil: Sollten diese Daten irgendeinen Steuerungseffekt auslösen, dann dürfte wohl mehr Zeit in die Patientenselektion und in die Beschönigung der Zahlen investiert werden als in wirkliche Qualitätsverbesserungsmassnahmen.

\section{Wir Ärztinnen und Ärzte müssen unsere Qualitätsaktivitäten aktiv gegen aussen tragen}

Die schlechte Qualität der BAG-Mortalitätszahlen fordert gleichzeitig uns Ärztinnen und Ärzte auf, unsere Qualitätsaktivitäten aktiver gegen aussen zu tragen und mitzuhelfen, sinnvolle Indikatoren und Methoden zur Qualitätserhebung zu definieren. Wenn die Mortalitätsstatistik des Bundes etwas gebracht hat, dann die Erkenntnis, dass ohne unser Wissen, das aus der täglichen Arbeit mit dem Patienten entsteht, kein echter Fortschritt in der Qualitätsdiskussion zu erreichen ist.

Dr. med. Daniel Herren, MHA, Mitglied des Zentralvorstandes der FMH, Verantwortlicher für das Ressort DDQ 